\title{
TWO TYPES OF SOLUTION OVERSHOOTS IN DISCONTINUOUS GALERKIN DISCRETIZATION SCHEMES*
}

\author{
N. B. PETROVSKAYA ${ }^{\dagger}$
}

Abstract. We consider high order Discontinuous Galerkin (DG) discretization for steady state problems. It will be demonstrated that using a high order DG scheme to discretize a problem may result in two types of solution overshoots. The oscillations of the first type are associated with smooth approximation of solution discontinuities. In addition, the oscillations may appear in steady state problems as a result of incorrect flux approximation near the flux extremum point.

Key words. discontinuous Galerkin, steady state problem, spurious oscillations

AMS subject classifications. 49M15, 65L20, 65L60

\section{Introduction}

Over last three decades Discontinuous Galerkin (DG) numerical schemes have been intensively investigated by many authors (see [5] for the review of DG schemes). The interest to the DG schemes is based on their many advantages, one of which is that the optimal orders of convergence may be achieved by using high order approximating spaces. However, the implementation of high order DG schemes for real - life applications is a complex problem, which usually requires a significant computational effort. This makes the choice of a numerical strategy a demanding issue, so that a taken decision should be based on clear understanding of the discretization features. In consideration of high order DG schemes there are a number of works where the authors support the following statements.

(i) Oscillations in high order DG schemes are due to solution approximation near discontinuities and may be cured either by using limiters or by adding some stabilization terms (e.g. artificial dissipation) to the scheme. This widespread opinion is supported in $[3,6,10,11,14,17,21]$.

(ii) From a convergence point of view, all numerical fluxes can be equally used for high order DG schemes. It has been pointed out in the work [4] that "...numerical experience suggests that as the degree $\mathrm{k}$ of the approximate solution increases, the choice of the numerical flux does not have a significant impact on the quality of the approximation." The similar point of view has been conducted in $[19,21]$. Thus, in many cases practitioners use a numerical flux which choice is based on computational aspects (e.g. computational cost of the flux, how far the flux definition is complicated to code it, etc.) rather than the properties of the flux itself (e.g. see $[7,10,17,18]$ ).

While the above-mentioned point of view is mainly based on the successful implementation of DG schemes the for numerical solution of hyperbolic systems of conservation laws $[4,7,17,18,19]$, in the present paper an attempt has been made to discuss the issues (i), (ii) for steady state problems. A discretization of steady state problems is often associated with the numerical solution of conservation laws, as a widely adopted strategy is to use a time dependent algorithm to approach a steady state. Alternatively, we address a high order DG scheme coupled with Newton's method as

\footnotetext{
${ }^{*}$ Received: August 20, 2004; accepted (in revised version): April 16, 2005. Communicated by Shi Jin.

${ }^{\dagger}$ School of Mathematics, the University of Birmingham, Edgbaston, Birmingham, B15 2TT, United Kingdom (n.b.petrovskaya@bham.ac.uk).
} 
an approach suggesting potential computational benefits in a numerical solution of steady state problems. Since this approach has not been well studied yet, ordinary differential equations are considered which provide us with a simple but illustrative model of a steady state problem.

In our work, we identify a possible origin of solution oscillations arising as a result of a high order DG discretization of a steady state problem. It will be shown that the high order DG scheme may result in two types of solution overshoots. The oscillations of the first type are associated with smooth approximation of solution discontinuities. The results of the paper confirm the statement (i) by demonstration that even in the simplest case of a linear ODE one may expect a solution overshoot, as a discontinuous solution is approximated by smooth functions in a high order DG scheme. The oscillations of the second type may appear in steady state problems as a result of incorrect flux approximation near a flux extremum point. We demonstrate that incorrect flux approximation impacts on the iterative method used in the problem (e.g. Newton's method) resulting in oscillations that cannot be eliminated by local limiters. This result indicates that the choice of a numerical flux should be more carefully advocated, and the statement (ii) requires a closer look when dealing with high order DG schemes.

\section{The solution approximation for high order DG schemes}

In this section we discuss solution oscillations arising as a result of approximation to a discontinuous solution by smooth functions. We consider an ordinary differential equation written for a function $u(x)$ as

$$
F_{x}(x, u)=S(x), \quad x \in \Omega=[a, b]
$$

where, on the analogy with a conservation law, the function $F(x, u(x))$ is thought of as a flux function. An appropriate boundary condition will be further provided for the equation (2.1) for a given problem under consideration.

In a DG method, a weak formulation of the problem is used to find the approximate solution $u_{h}(x)$, since the solution $u(x)$ to the problem (2.1) may be a discontinuous function. We introduce a computational grid as $G=\bigcup_{i=1}^{N} e_{i}, e_{i}=\left[x_{i}, x_{i+1}\right], 1 \leq i \leq N$, where $x_{i}$ is a nodal coordinate, and $h_{i}=x_{i+1}-x_{i}$ is a grid step size. The notation $x_{i}-0$ and $x_{i}+0$ is used for the left and right limits at the point $x_{i}$. The test functions $\phi_{k}(x)$ are defined on the grid cell $e_{i}$ for $k=0,1, \ldots, K$ as

$$
\phi_{k}(x)=\left(\frac{x-x_{i}}{h_{i}}\right)^{k}, x \in e_{i}
$$

Multiplying the equation (2.1) by test function $\phi_{k}(x)$ and integrating by parts over the cell $e_{i}$ gives

$$
\begin{gathered}
F\left(x_{i+1}, u\left(x_{i+1}\right)\right) \phi_{k}\left(x_{i+1}\right)-F\left(x_{i}, u\left(x_{i}\right)\right) \phi_{k}\left(x_{i}\right)- \\
\int_{x_{i}}^{x_{i+1}} F(x, u) \frac{d \phi_{k}(x)}{d x} d x=\int_{x_{i}}^{x_{i+1}} S(x) \phi_{k}(x) d x, \quad k=0,1, \ldots, K
\end{gathered}
$$

where the function $u(x)$ should be further replaced by the approximate solution $u_{h}(x)$. 
The approximation $u_{h}(x)$ to the solution $u(x)$ in the DG scheme is a piecewise polynomial function over the domain $\Omega$,

$$
u_{h}(x)=\sum_{k=0}^{K} u_{k} \phi_{k}(x), \quad k=0,1, \ldots, K, \quad x \in e_{i} .
$$

Since $u_{h}(x)$ is discontinuous at grid nodes, the equation (2.2) requires flux approximation at any grid interface. An approximate Riemann solver elaborated in order to discretize the flux in the equation $(2.2)$ results in a numerical flux $\tilde{F}\left(u_{h}\right)$, which generally depends on the two values of the approximate solution at any grid node $x_{i}$. In our work we use the Engquist -Osher definition [8] of the numerical flux

$$
\tilde{F}^{E O}\left(u_{h}\right)=\int_{0}^{u_{r}} \min \left(F^{\prime}(s), 0\right) d s+\int_{0}^{u_{l}} \max \left(F^{\prime}(s), 0\right) d s+F(0),
$$

where $u_{l}=u_{h}\left(x_{i}-0\right)$ and $u_{r}=u_{h}\left(x_{i}+0\right)$ are left and right solution values at the grid node $x_{i}$, respectively.

After the flux $\tilde{F}\left(u_{h}\right)$ is defined for a given problem, the DG discretization scheme reads

$$
\begin{gathered}
\tilde{F}\left(u_{h}\left(x_{i+1}\right)\right) \phi_{k}\left(x_{i+1}\right)-\tilde{F}\left(u_{h}\left(x_{i}\right)\right) \phi_{k}\left(x_{i}\right)- \\
\int_{x_{i}}^{x_{i+1}} F\left(x, u_{h}(x)\right) \frac{d \phi_{k}(x)}{d x} d x=\int_{x_{i}} S(x) \phi_{k}(x) d x, \quad k=0,1, \ldots, K .
\end{gathered}
$$

We begin our discussion with consideration of a simple linear boundary - value problem

$$
u_{x}=S(x), \quad u(0)=U_{0}, \quad x \in \Omega=[0,2],
$$

with flux function $F(x, u) \equiv u$. The appearance of solution overshoots near a discontinuity point is well illustrated by consideration of the linear problem (2.6), as one can easily obtain analytic expression for the DG approximate solution when a discontinuous solution defined by $S(x)$ is discretized. Thus, a special $\mathrm{S}(\mathrm{x})$ will be chosen on the right-hand side in order to simulate a discontinuous solution to the problem (2.6).

The following numerical test has been considered in [20] to study a high order DG discretization for the linear problem (2.6). Let a discontinuous solution to the problem (2.6) be

$$
U(x)=\left\{\begin{array}{cc}
1 .-\sqrt{0.5-x} & 0 \leq x<0.5 \\
1 & 0.5<x \leq 1 \\
\tanh (200(x-1.5)) & 1<x \leq 2
\end{array}\right.
$$

The solution $U(x)$ is shown as a dashed curve in fig.2.1. The function $U(x)$ is discontinuous at $x_{s h}=1$ and has a discontinuous derivative at $x_{d}=0.5$. It also has a "boundary layer", i.e. a steep solution gradient, at the vicinity of the point $x_{b}=1.5$.

Let us now look at the numerical solution to the problem (2.6). For the model function $U(x)$ under consideration, the source function $S(x)$ is defined by straightforward differentiation of the solution. Namely, based on our knowledge of the solution $U(x)$ we discretize the source function as $S(x) \equiv d U(x) / d x$ to simulate a discontinuous solution. 

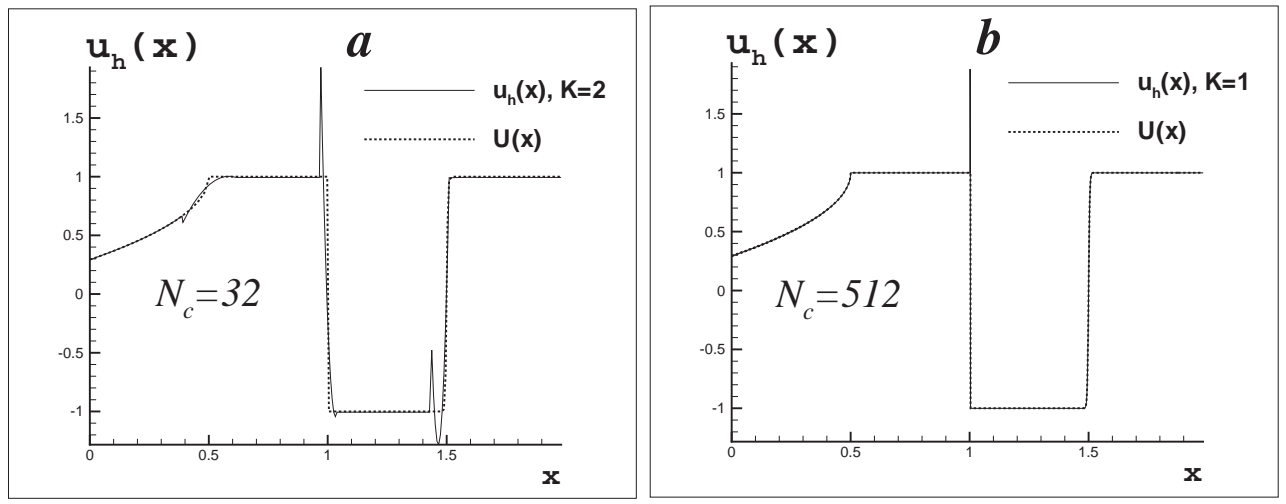

FIG. 2.1. High order DG solution $u_{h}(x)$ of a linear boundary - value problem (2.6). (a) Solution oscillations on a coarse grid for the polynomial degree $K=2$. (b) Solution oscillations near a discontinuity on a fine grid for the polynomial degree $K=1$.

A high order $(K>0)$ DG scheme has been used to discretize the problem (2.6). Since for the advection equation (2.6) we have $F(x, u) \equiv u$, the definition of the numerical flux (2.4) applied to the problem results in a well known upwind scheme with $\tilde{F}\left(u_{h}\right)=u_{l}$. Nonuniform grids have been generated in our numerical tests in order to prevent a location of the solution singularities at grid nodes.

The results of numerical experiments with the problem are shown in fig.2.1. An example of the approximate solution on a coarse grid is given by fig.2.1a, where the number of grid cells is $N_{c}=32$ and the polynomial degree of the DG discretization is $K=2$. It can be seen from the figure that the numerical solution has an overshoot on each cell where a singularity is present and it oscillates at the boundary layer as well. Further, the approximated solution is resolved on fine grids in the boundary layer region. The oscillations at the shock, however, stay on fine grids for any polynomial degree $K>0$. An example of the approximate solution on a fine grid with $N_{c}=512$ cells is shown for the polynomial degree $K=1$ in fig.2.1b.

2.1. High order DG approximation at the shock for a linear problem.

The solution overshoots at the shock can be easily explained by considering the step function as a solution to the problem (2.6). We now solve (2.6) at the segment $[0,1]$ and seek for the following discontinuous solution

$$
U^{s t}(x)=\left\{\begin{array}{l}
U_{0}, 0 \leq x<x_{s h} \\
U_{1}, x_{s h} \leq x \leq 1
\end{array}\right.
$$

where $x_{s h}$ is a shock position within a grid cell $e_{i}, x_{i}<x_{s h}<x_{i+1}$. Without loss of generality, we further consider $U_{0}=0, x_{i}=0$, and $x_{i+1}=h$. A set of basis functions on the cell $e_{i}$ is now given by $\phi_{k}(x)=x^{k}, \quad k=0,1, \ldots, K$, and the shock position $x_{s h}$ is parametrized by

$$
x_{s h}=\alpha h, \quad 0<\alpha<1 .
$$

The source $S(x)=d U^{s t}(x) / d x$ can be formally considered as $S(x)=C \delta\left(x-x_{s h}\right)$, 
where $\delta(x)$ is the Dirac $\delta$-function defined by

$$
\int_{\xi_{1}}^{\xi_{2}} \delta\left(\xi-\xi^{*}\right) f(\xi) d \xi=\left\{\begin{array}{cc}
f\left(\xi^{*}\right), & \xi^{*} \in\left[\xi_{1}, \xi_{2}\right], \\
0, & \xi^{*} \notin\left[\xi_{1}, \xi_{2}\right] .
\end{array}\right.
$$

The value of the scaling factor $C$ is obtained by integrating over the interval $[0, h]$,

$$
\int_{0}^{h} S(x) d x=C \int_{0}^{h} \delta\left(x-x_{s h}\right) d x=C .
$$

On the other hand, we have

$$
\int_{0}^{h} S(x) d x=\int_{0}^{h} \frac{d U(x)}{d x} d x=U(h)-U(0)=U_{1}-U_{0},
$$

so that the scaling factor is $C=[u]$, where $[u]=U_{1}-U_{0}$ is the solution jump amplitude at the point $x_{s h}$.

As an example of high order DG discretization we consider a linear reconstruction $(K=1)$ of the discontinuous solution $(2.7)$ on the cell $e_{i}$

$$
u_{h}(x)=u_{0}+u_{1} x .
$$

The definition of the numerical flux requires replacing the value $u_{h}(0)$ by the boundary value $U_{0}$. Substituting (2.9) into the system (2.5) and taking into account $U_{0}=0$ yields the following system for the expansion coefficients $\left(u_{0}, u_{1}\right)$ on the cell where the shock is present

$$
\begin{gathered}
u_{0}+u_{1} h=\int_{0}^{h} S(x) d x \\
\left(u_{0}+u_{1} h\right) h-\int_{0}^{h}\left(u_{0}+u_{1} x\right) d x=\int_{0}^{h} S(x) x d x .
\end{gathered}
$$

Taking into account the parametrization (2.8), the right-hand side integrals in (2.10) are as follows

$$
\int_{0}^{h} S(x) d x=[u], \int_{0}^{h} S(x) x d x=\alpha h[u] .
$$

Solving the system (2.10) with the right-hand side (2.11) gives us the following values of the expansion coefficients in $(2.9)$

$$
u_{0}=(1-2 \alpha)[u], \quad u_{1}=2 \alpha \frac{[u]}{h} .
$$

It follows from the first equation in (2.10) that the DG solution $u_{h}(x)$ at the right boundary of the cell is $u_{h}(h)=u_{0}+u_{1} h=\int_{0}^{h} S(x) d x=U^{s t}(h)=U_{1}$. Furthermore, the 
linear solution (2.9) is a monotone function over the grid cell. Thus, the only point where a solution overshoot may occur is the "inflow" boundary $x=0$.

The DG solution at the left boundary is $u_{h}(0)=u_{0}$. It follows from (2.12) that the value $u_{0}$ in the expansion (2.9) does not depend on the element size $h$. The approximate solution at the left boundary depends only on the shock position relative to the right boundary, $u_{0}=u_{0}(\alpha)$. Since the solution error at the left boundary is

$$
\left|U(0)-u_{h}(0)\right|=\left|u_{0}(\alpha)\right|=\left|(1-2 \alpha) U_{1}\right| \neq 0, \quad \forall \alpha \neq 1 / 2
$$

the approximate solution obtained as a result of the DG discretization does not converge in the norm $L_{\infty}=\max _{x \in e_{i}}\left|U(x)-u_{h}(x)\right|$ on the interval $[0, h]$.

The parameter $\alpha$ generates the solution field $u_{h}(x, \alpha)$. It is readily seen from (2.12) that the value $\alpha_{0}=1 / 2$ is the only shock location which provides the solution $u_{h}\left(x, \alpha_{0}\right)$ with no jump at the inflow boundary. For any value $\alpha \neq \alpha_{0}$, the approximate solution remains discontinuous at the inflow boundary, as $h \rightarrow 0$. Hence, the critical value $\alpha_{0}$ can be obtained from the continuity requirement $u\left(0, \alpha_{0}\right)=U_{0}$, and a linear DG discretization of the discontinuous solution can be considered as a shock transfer from point $x_{s h}$ to the inflow boundary $x=0$.

The amplitude of a discontinuity at the left boundary generated by the DG discretization depends on the original shock location. For the shock stationed at $0<\alpha \leq \alpha_{0}$, no overshoot of the solution occurs at the left boundary, since the value $u_{0}(\alpha)$ is bounded by $0 \leq u_{0}(\alpha)<U_{1}$. Thus any approximate solution (2.9) generated by parameter $0<\alpha \leq \alpha_{0}$ is acceptable in the sense that $u_{h}(x)$ is a monotone function and $u_{h}(x)$ has a variation within true solution limits. On the contrary, any value $\alpha_{0}<\alpha<1$ generates a solution overshoot at the inflow boundary. This overshoot is illustrated for the linear DG discretization in fig.2.1b, where the shock is stationed on the cell in such a way that $\alpha=0.83$ on a fine grid.

The approach similar to that discussed above can be applied to the solution approximation with an arbitrary polynomial degree $K>0$. The system (2.10) now consists of $K+1$ equations, and the value $u_{0}$, obtained as a result of solving (2.10), provides us with the amplitude of a solution jump at the interface. The solution jump is a function of the shock location $\alpha$ and it does not depend on a grid step size. However, the solution field $u_{h}(x, \alpha)$ now has a more complicated structure in comparison with that obtained in the linear case. Generally, an approximate solution may have $K$ extremum points in the domain $e_{i}$. Those extrema should be also taken into account in consideration of solution overshoots, as they keep the approximate solution nonmonotone on the cell where a discontinuity is present. Again, the solution extrema do not disappear on refined grids, since their appearance depends only on the shock location.

2.2. High order DG approximation at the shock for a nonlinear problem. For a nonlinear steady state problem it is difficult to derive a general expression for DG approximation to a discontinuous solution. However, based on the results obtained for a linear problem, one may expect a solution overshoot near a discontinuity when the DG solution with a polynomial degree $K>0$ is considered.

The following example illustrates high order DG approximation for a nonlinear problem. Consider a boundary-value problem known as the problem of mass flow in a convergent - divergent nozzle, [1]. Let $\mathrm{A}(\mathrm{x})$ be the area of the nozzle, $A(x)=1 / 2+$ $2(x-1 / 2)^{2}, \quad 0 \leq x \leq 1$, and $u(x)$ be the velocity deviation. The mass conservation in 

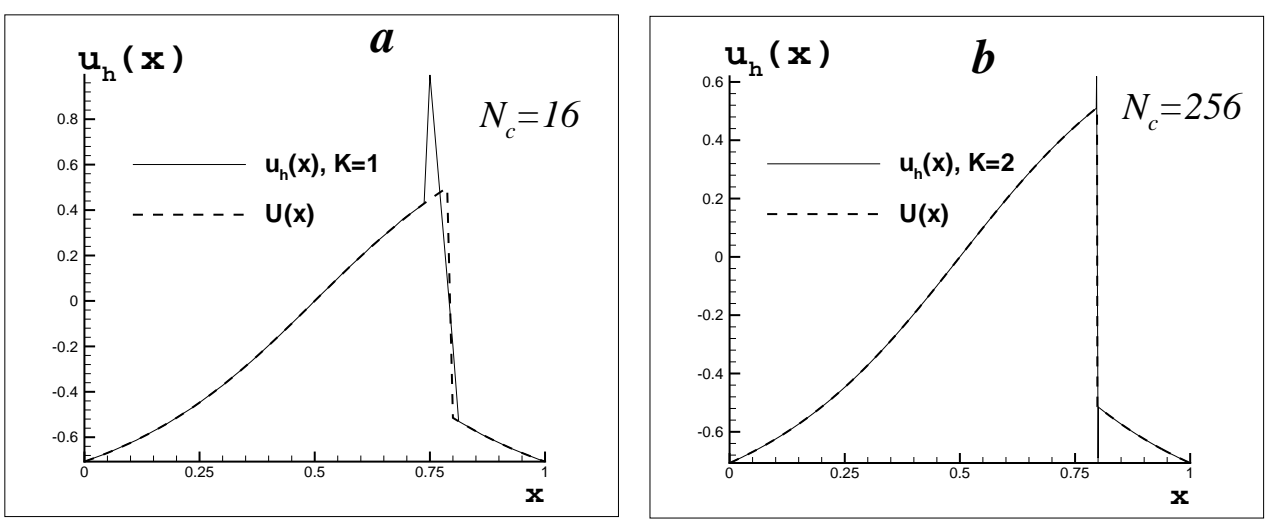

FIG. 2.2. Numerical solution to nonlinear boundary - value problem (2.13), (2.15). Solution oscillations near a discontinuity. The true solution to the problem is shown as a dashed line. $(a)$. A piecewise linear solution reconstruction $(K=1)$ leads to the solution overshoot on the cell where a discontinuity is present. (b). Solution oscillations on a fine grid for a DG discretization with polynomial degree $K=2$.

the nozzle can be modelled as

$$
\frac{d F(x, u(x))}{d x} \equiv \frac{d(A(x) m(u))}{d x}=0, \quad x \in[0,1],
$$

where the mass flux through the nozzle is given by

$$
m(u)=\frac{1}{2}\left(1-u^{2}\right) .
$$

The equation (2.13) is solved due to the boundary condition

$$
\int_{0}^{1} u(x) d x=B
$$

where $B$ is a constant which determines the solution type. The choice $B=-0.25$ allows one to obtain a discontinuous solution

$$
U(x)=\left\{\begin{array}{c}
-\sqrt{(1-1 / 2 A(x))}, \quad 0 \leq x \leq x_{s}, \text { or } x_{s h}+0 \leq x \leq 1, \\
\sqrt{(1-1 / 2 A(x))}, \quad x_{s} \leq x \leq x_{s h}-0,
\end{array}\right.
$$

where $x_{s h}$ is a shock location, and $x_{s}: u\left(x_{s}\right)=u_{s}=0$ is stated as $x_{s}=1 / 2$. The value $u=0$ is a flux extremum point. The solution $U(x)$ is shown in fig.2.2.

Integrating the discontinuous solution $U(x)$ over the domain $[0,1]$ yields the following algebraic equation with respect to the variable $x_{s h}$

$$
I_{1}+I_{2}\left(x_{s h}\right)+I_{3}\left(x_{s h}\right)=B
$$

where $\quad I_{1}=-\int_{0}^{x_{s}} \sqrt{1-1 / 2 A(x)} d x, \quad I_{2}\left(x_{s h}\right)=\int_{x_{s}}^{x_{s h}} \sqrt{1-1 / 2 A(x)} d x, \quad$ and $\quad I_{3}\left(x_{s h}\right)=$ $-\int_{x_{s h}}^{1} \sqrt{1-1 / 2 A(x)} d x$. Solving this equation for a given value of $B$, the shock location can be defined. For $B=-0.25$, the shock will be located at $x_{s h}=0.798074$. 
We use the definition (2.4) of the numerical flux in the numerical solution of the problem. Given the left and right state at the interface, the Engquist-Osher numerical flux is as follows

$$
\tilde{m}\left(u_{l}, u_{r}\right)=\left\{\begin{array}{l}
m\left(u_{r}\right), \quad u_{l}<0, u_{r}<0, \\
m\left(u_{l}\right), \quad u_{l}>0, u_{r}>0 \\
m(0), \quad u_{l}<0, u_{r}>0, \\
m\left(u_{l}\right)+m\left(u_{r}\right)-m(0), \quad u_{l}>0, u_{r}<0 .
\end{array}\right.
$$

The DG space discretization of the nonlinear problem (2.13) results in the following system of nonlinear equations

$$
\mathbf{R}(\mathbf{u})=0,
$$

where the vector $\mathbf{R}(\mathbf{u})$ is the residual of the DG method given by (2.5) and the discretization of boundary condition (2.15), and $\mathbf{u}$ is the solution vector. We use Newton's iteration method to solve the nonlinear equations (2.18). Let $\mathbf{u}^{n}$ and $\mathbf{u}^{n+1}$ be the solution vector at $n$-th and $n+1$-th solution iteration, respectively. Then the linearized system is

$$
\mathbf{J}\left(\mathbf{u}^{n}\right)\left(\mathbf{u}^{n+1}-\mathbf{u}^{n}\right)=-\mathbf{R}\left(\mathbf{u}^{n}\right)
$$

where the Jacobian matrix $\mathbf{J}(\mathbf{u})=[\partial \mathbf{R} / \partial \mathbf{u}]$ and residual $\mathbf{R}(\mathbf{u})$ are taken from the $n$-th iteration. The GMRES algorithm ([16], [2]) is used to solve numerically a system of linear equations obtained at each Newton's iteration.

In order to illustrate the approximation of the discontinuous solution by a high order DG scheme we choose the true solution (2.16) as an initial guess for Newton's iterations. The initial guess is discretized by solving

$$
\int_{x_{i}}^{x_{i+1}} u_{h}(x) \phi_{k}(x) d x=\int_{x_{i}}^{x_{i+1}} U(x) \phi_{k}(x) d x, \quad k=0,1 \ldots, K,
$$

where the approximate solution $u_{h}(x)$ is given by (2.3) on each grid cell. A piecewise polynomial function $u_{h}^{0}(x)$ obtained as a result of the above discretization coincides with the exact solution $U(x)$ at any grid node and has no jumps at grid interfaces. However, the solution approximation does not meet the condition

$$
\|\mathbf{R}(\mathbf{u})\|=\max _{i=1, \ldots, N}|\mathbf{R}(\mathbf{u})|<\epsilon
$$

which is used as a convergence criterion for Newton's iterations.

Let us generate a computational grid in such a way that the solution discontinuity is located at an interior point of the grid cell $e_{s h}$. After a few Newton's iterations the residual norm rapidly decreases to a small value $\epsilon=1.0 e-16$, which indicates the convergence of the method. However, the approximate solution with polynomial degree $K>0$ does not coincide with the true solution at the grid nodes anymore, as it now has an overshoot on a cell where a discontinuity is present. An example of the approximate solution with polynomial degree $K=1$ is shown in fig.2.2a on a coarse grid of $N_{c}=16$ cells. Furthermore, as one can expect from the analysis of the linear problem, the solution overshoots will appear on fine grids as well, if we use the same approach to obtain a numerical solution. The solution on a fine grid is shown in 
fig.2.2b where the number of grid cells is $N_{c}=256$ and a polynomial degree is chosen $K=2$.

Solution overshoots arising as a result of the inconsistent approximation of a discontinuity need to be eliminated, as they may slow down the convergence of the Newton method by moving a transient solution out of the basin of attraction. Once the solution overshoot has been detected, a simple way to control the oscillations is to decrease the solution to a piecewise constant reconstruction at the discontinuity at the current Newton step. However, our numerical experience with steady state problems reveals that simple reducing the polynomial degree is not sufficient to obtain a convergent solution. In addition to the solution overshoots due to the smooth approximation of a discontinuity, another type of oscillation may occur in numerical solution of steady state problems. Below we discuss the second type of solution oscillations which appeared to be dangerous for the Newton method.

\section{The numerical flux for high order schemes}

For the oscillations of the second type we consider the nonlinear problem (2.13), (2.15) which is now solved by Newton's method starting with an arbitrary initial guess. Namely, we seek a numerical solution to the problem on a sequence of uniform grids. Each following grid in the sequence is refined by cutting grid cells into halves. The initial guess on the first grid is chosen as $u_{0}(x)=$ const $=-1.0$. The initial guess for the next finer grid is obtained by linear interpolation of the numerical solution taken from a previous grid.

The above approach is supposed to provide the fast convergence of the Newton method, as it makes the initial guess close to the solution on each finer grid. Nevertheless, our numerical experiments show that the Newton method fails to obtain a convergent solution for high order DG discretization. Only a piecewise constant discretization $K=0$ reconstructs the discontinuous solution $U(x)$ on a sequence of grids. Meanwhile, any DG discretization with polynomial degree $K>0$ yields spurious oscillations on the first grid in the sequence. Those oscillations lead to a divergent solution on the initial grid so that calculations on finer grids become impossible. The evolution of solution oscillations on the initial grid is presented in fig.3.1 where transient solutions are shown for several first Newton's iterations. In the figure, a transient solution $u^{I}$ is the initial guess for the method, $u^{I}=$ const $=-1.0$, and the Roman notation is used to mark the Newton iterations.

On the surface, a reasonable explanation of the spurious oscillations displayed in the figure is that once a solution overshoot discussed in the previous sections has appeared near the discontinuity, the oscillations propagate over the domain of computation resulting in a divergent solution. Nevertheless, the nature of oscillations is different from that considered in section 2. It can be seen from the figure that the solution overshoots first arise before a shock formation. Actually, let us consider two transient solutions $u^{I I}$ and $u^{I I I}$ shown in the figure. Both of them can be considered as "smooth" functions in the sense that they can be interpolated by a smooth continuous curve. Using the transient solution $u^{I I}$ as the entering data for the third Newton iteration yields the solution $u^{I I I}$ which is a non-oscillating function. However, entering the next Newton iteration with the smooth solution $u^{I I I}$ results in spurious oscillations in the function $u^{I V}$.

A difference between the functions $u^{I I}$ and $u^{I I I}$ is that for the transient solution $u^{I I}$ the flux $F(u)$ is a monotone function over the domain of definition $u_{h}(x) \in\left[u_{\min }^{I I}, u_{\max }^{I I}\right]$. It can be seen from the figure that the solution $u^{I I}$ lies below the flux extremum $u=0$. Meanwhile, for the function $u^{I I I}$, we have $u_{\min }^{I I I}<0$, 


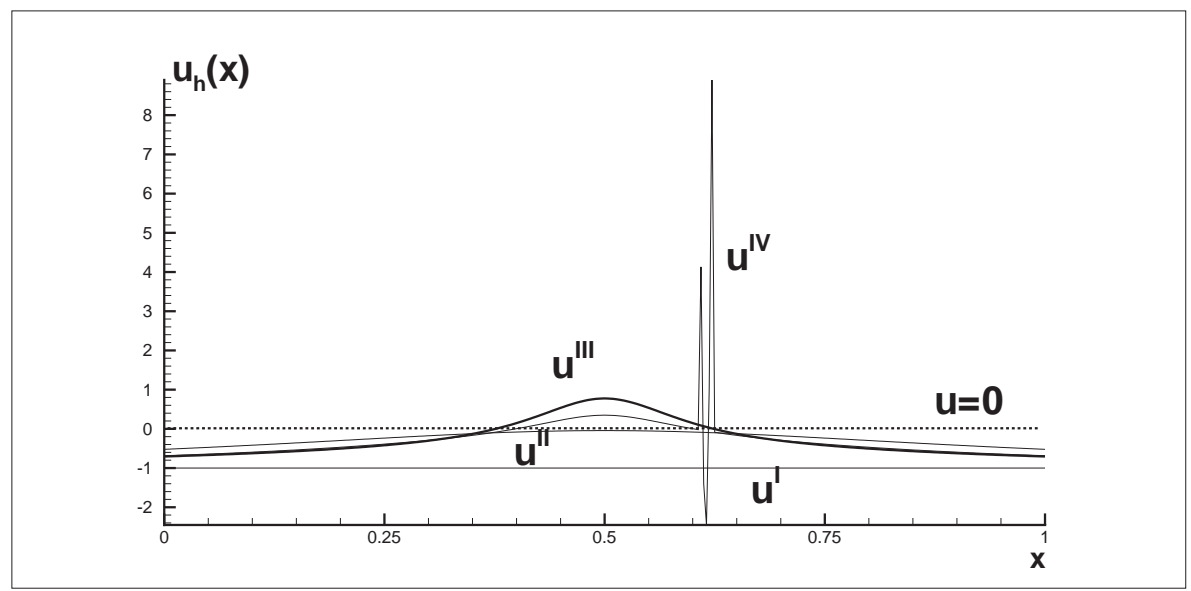

FIG. 3.1. Numerical solution of the problem (2.13), (2.15) on the initial grid. Appearance of oscillations in Newton's method for a high order DG scheme $K>0$. Oscillations arise in transient solution $u^{I V}$ due to the flux approximation at the extremum point $u=0$ at the previous Newton iteration where transient solution $u^{I I I}$ is involved.

$u_{\text {max }}^{I I I}>0$, so that we need to approximate the flux at the extremum point $u=0$, as it is required by the DG formulation in order to obtain the transient solution $u^{I V}$.

The flux approximation in the DG scheme can be best illustrated by consideration of the DG equations on a single cell $e_{i}$. For the sake of the further discussion, let us suppose that the approximate solution on adjacent cells $e_{i-1}$ and $e_{i+1}$ is already determined. Due to a compact stencil of the DG discretization, this approach can be considered as the numerical solution of the equation (2.13) in the domain $\Omega=e_{i}$ subject to the boundary conditions required to determine fluxes in the DG scheme.

Let $U_{1}=u_{h}\left(x_{i}-0\right)$ be the left solution state at the interface $x_{i}$ and $U_{2}=u_{h}\left(x_{i+1}+\right.$ 0 ) be the right solution state at the interface $x_{i+1}$. The values $U_{1}$ and $U_{2}$ are known, as they are taken from the solution on the adjacent cells. Let us also denote the solution values at the interfaces of the cell $e_{i}$ as $u^{l}=u_{h}\left(x_{i}+0\right)$ and $u^{r}=u_{h}\left(x_{i+1}-0\right)$, respectively. We notice that, unlike the flux definition (2.17), the solution states $u^{l}$ and $u^{r}$ are now associated with different cell interfaces. The values $u^{l}$ and $u^{r}$ are related to the unknowns $\left(u_{0}, u_{1}, u_{2}, \ldots, u_{K}\right)$ on the cell $e_{i}$ as follows

$$
u^{l}=u_{0}, \quad u^{r}=\sum_{k=0}^{K} u_{k} .
$$

According to (2.2), the DG equations on the cell $e_{i}$ are as follows

$$
\begin{gathered}
\tilde{F}\left(u_{h}\left(x_{i+1}\right)\right) \phi_{k}\left(x_{i+1}\right)-\tilde{F}\left(u_{h}\left(x_{i+1}\right)\right) \phi_{k}\left(x_{i+1}\right)=0, \\
\tilde{F}\left(u_{h}\left(x_{i+1}\right)\right) \phi_{k}\left(x_{i+1}\right)-\frac{1}{h_{i}} \int_{x_{i}} F\left(x, u_{h}\right) d x=0, \\
\vdots \\
\tilde{F}\left(u_{h}\left(x_{i+1}\right)\right) \phi_{k}\left(x_{i+1}\right)-\frac{K}{h_{i}^{K}} \int_{x_{i}}^{x_{i+1}} F\left(x, u_{h}\right)\left(x-x_{i}\right)^{K-1} d x=0 .
\end{gathered}
$$


Let the flux $F(u)$ be a monotone function over the domain $e_{i}$. Then the numerical flux is given by

$$
\tilde{F}\left(u_{h}\left(x_{i}\right)\right)=F\left(x_{i}, u^{l}\right), \quad \tilde{F}\left(u_{h}\left(x_{i+1}\right)\right)=F\left(x_{i+1}, U_{2}\right),
$$

for an increasing flux function, $F^{\prime}(u)>0, u \in\left[U_{1}, U_{2}\right]$. For a decreasing flux function, $F^{\prime}(u)<0, u \in\left[U_{1}, U_{2}\right]$, we have

$$
\tilde{F}\left(u_{h}\left(x_{i}\right)\right)=F\left(x_{i}, U_{1}\right), \quad \tilde{F}\left(u_{h}\left(x_{i+1}\right)\right)=F\left(x_{i+1}, u^{r}\right) .
$$

Substituting the above numerical flux into (3.2) results in a system of nonlinear algebraic equations with respect to variables $\left(u_{0}, u_{1}, \ldots, u_{K}\right)$ which define the approximate solution $u_{h}$ on the cell $e_{i}$. Let us notice that due to (3.1) each equation in (3.2) involves the variables $\left(u_{0}, u_{1}, \ldots, u_{K}\right)$. Hence, for a monotone flux function we have $K+1$ unknowns on the cell $e_{i}$ and $K+1$ equations to be solved.

We now look at a nonmonotone flux on the cell $e_{i}$. Suppose that the flux extremum appears at the point $x=x^{*}, u=u^{*}$ within the cell $e_{i}$, where we have $u^{*}=0$ for the problem (2.13). Let us consider $u^{l}>0$ and $u^{r}<0$. Since $\left(x^{*}, u^{*}=0\right)$ is an interior point of the cell $e_{i}$, we also have $U_{1}>0$ and $U_{2}<0$. Then, according to the flux definition (2.17), the numerical flux is defined as

$$
\tilde{F}\left(u_{h}\left(x_{i}\right)\right)=F\left(x_{i}, U_{1}\right), \quad \tilde{F}\left(u_{h}\left(x_{i+1}\right)\right)=F\left(x_{i+1}, U_{2}\right),
$$

and the discrete conservation law, i.e. the first of the DG equations (3.2), reads

$$
F\left(x_{i+1}, U_{2}\right)-F\left(x_{i}, U_{1}\right)=0 .
$$

Since the above equation does not contain any of the unknowns $\left(u_{0}, u_{1}, \ldots, u_{K}\right)$, the number of unknowns exceeds the number of equations in (3.2). Hence, the system of DG equations is underdetermined for a nonmonotone flux function on the cell $e_{i}$.

Solving the underdetermined system (3.2) presents us with certain difficulties, as the solution to such a system is not unique. In particular, considering the Newton method, one may expect a singular Jacobian in the problem. Namely, let us linearize the DG equations on the cell $e_{i}$. For the first equation in (3.2), the Jacobian entries $j_{1 k}$ are defined as follows

$$
j_{1 k}=\frac{\partial R_{1}}{\partial u_{k}}=\frac{\partial}{\partial u_{k}}\left(\tilde{F}\left(u_{h}\left(x_{i+1}\right)\right) \phi_{k}\left(x_{i+1}\right)-\tilde{F}\left(u_{h}\left(x_{i+1}\right)\right) \phi_{k}\left(x_{i+1}\right)\right),
$$

where the derivatives are

$$
\frac{\partial \tilde{F}\left(u_{h}\right)}{\partial u_{k}}=\left(\frac{\partial \tilde{F}\left(u_{h}\right)}{\partial u^{l}}\right) \frac{\partial u^{l}}{\partial u_{k}}+\left(\frac{\partial \tilde{F}\left(u_{h}\right)}{\partial u^{r}}\right) \frac{\partial u^{r}}{\partial u_{k}}, \quad k=0,1, \ldots K .
$$

Since the numerical flux in the equation (3.3) does not depend on $u^{l}$ and $u^{r}$, we have

$$
\frac{\partial F\left(x_{i}, U_{1}\right)}{\partial u^{l}}=0, \quad \frac{\partial F\left(x_{i+1}, U_{2}\right)}{\partial u^{l}}=0, \quad \frac{\partial F\left(x_{i}, U_{1}\right)}{\partial u^{r}}=0, \quad \frac{\partial F\left(x_{i+1}, U_{2}\right)}{\partial u^{r}}=0,
$$

so that a row with zero entries appears in the Jacobian matrix.

Consequently, the singularity of the 'local' Jacobian on the cell $e_{i}$ affects the Newton method in the case that the linearized system (2.19) is solved as a result of the DG discretization over the grid. It has been proven in [15] that, due to the 
singular local matrix on the cell $e_{i}$, a zero column appears in the Jacobian of the system (2.19). The singular Jacobian in the method leads to an incorrect transient solution oscillating over the domain. Entering this solution as the initial data for the next Newton's iterations will result in the divergence of the method.

It follows from the above consideration that the result of the DG discretization depends strongly on the choice of the numerical flux in the problem. Generally, the definition of an upwind flux requires the solution of the Riemann problem at each grid interface, [12, 13]. It is well known that for a monotone flux function the solution to the Riemann problem involves just one solution state at the interface. Hence, if the solution variation $\delta u_{h}=u_{h}\left(x_{i+1}-0\right)-u_{h}\left(x_{i}+0\right)$ in a high order DG discretization generates a flux extremum at the interior point of the cell $e_{i}$, the flux will remain a monotone function at both interfaces $x_{i}$ and $x_{i+1}$, and the 'phantom' solution on the cell $e_{i}$ will not be involved in the flux definition in a high order DG scheme.

Meanwhile, space-centered fluxes usually require both solution states at the interface to approximate the flux. For instance, the local Lax - Friedrichs (L-F) flux

$$
\begin{gathered}
\tilde{F}^{L L F}\left(u_{l}, u_{r}\right)=\frac{1}{2}\left[F\left(u_{l}\right)+F\left(u_{r}\right)-C\left(u_{r}-u_{l}\right)\right], \\
C=\max _{\min \left(u_{l}, u_{r}\right) \leq s \leq \max \left(u_{l}, u_{r}\right)}\left|F^{\prime}(s)\right|,
\end{gathered}
$$

does not lead to an underdetermined system of the DG equations, as now both solution states at each interface are involved into the approximation. For the problem (2.13) considered on the cell $e_{i}$ the discrete conservation law reads

$$
A\left(x_{i+1}\right) \tilde{m}_{i+1}^{L L F}\left(u^{r}, U_{2}\right)-A\left(x_{i}\right) \tilde{m}_{i}^{L L F}\left(u^{l}, U_{1}\right)=0,
$$

where $\tilde{m}_{i}^{L L F}$ is the flux (3.4) at the interface $x_{i}$. It can be seen from the definition (3.4) that the discrete conservation law (3.5) now involves the unknowns $\left(u_{0}, u_{1}, \ldots, u_{K}\right)$ on the cell $e_{i}$.

On the other hand, it is well known $[9,13]$ that the L-F flux is more dissipative than upwind fluxes, so that the choice of the L-F flux may not be acceptable for a specific problem under consideration (e.g. see [19]). Besides, as a result of a dissipative nature of the L-F flux, an artificial boundary condition may be required when a high order DG scheme is used in the problem. Our numerical experience with the problem (2.13) has shown that the convergence of the Newton method turned out to be very sensitive to the choice of the artificial boundary condition. Thus the L-F flux needs careful study before concluding about its advantage over upwind fluxes which lead to an undetermined system of equations.

Let us mention again that the nature of oscillations arising as a result of the inconsistent flux approximation at the extremum is different from that has been discussed in the previous section. Our previous results only concerned the approximation of the solution function and implied a well defined numerical flux over a computational domain. Now the numerical flux $\tilde{F}(u)$ is not a correct approximation to the flux function $F(u)$ at the extremum point, although the solution may remain a smooth monotone function near the flux extremum. Also, it is important to notice that the oscillations are entirely due to a nonlinear nature of the problem (2.13). A linear problem (2.6), where the flux $F(u) \equiv u$ is a monotone function, remains free from that type of solution overshoots.

We now briefly discuss how to obtain a convergent solution to the problem (2.13), (2.15). The results of our study demonstrate that a DG discretization with polynomial degree $K>0$ will yield a solution overshoot on any grid cell where a discontinuity 

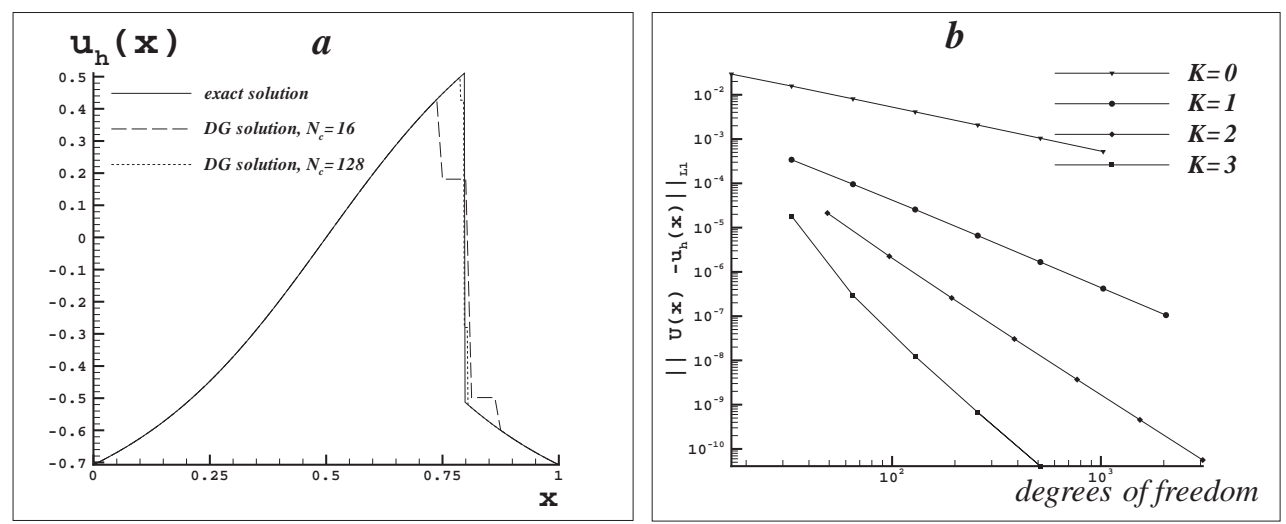

FIG. 3.2. Numerical solution to the problem (2.13), (2.15). (a) An example of the DG solution (polynomial degree $K=3$ ) on a coarse and fine grid. $N_{c}$ is the number of grid cells. (b) Convergence history for the DG solution with polynomial degree $K \geq 0$.

is present. However, simple reduction of the numerical solution to piecewise constant approximation near the shock to eliminate spurious solution oscillations is not successful in the problem and still results in a divergent solution. In addition, one should also take care of the flux approximation to avoid a singular Jacobian in the Newton method. Thus the algorithm of numerical solution of the problem (2.13), (2.15) developed in [15] seeks for nonphysical flux extrema within each grid cell in order to avoid a "phantom" solution on the cell. Once a "phantom" solution has been detected on the cell, the polynomial degree of the numerical solution is reduced on the cell to render the flux approximation correct. Our numerical experience with the problem shows that the control of each grid cell is absolutely required in the problem, as the domain of attraction for the Newton method may have a very complicated structure and nonphysical flux extrema may appear in a transient solution at each Newton iteration.

The control over the numerical flux to avoid a singular Jacobian and the control over the solution at the shock to avoid solution overshoots allow one to obtain convergent solutions for high order DG discretization schemes. The approximate DG solution with polynomial degree $K=3$ obtained by the Newton method is shown in fig.3.2a on a coarse uniform grid of 16 cells and fine grid of 128 cells. It can be seen from the figure that the shock in the numerical solution has to be smeared over two adjacent grid cells, as an uncorrected solution has an overshoot at the shock at the final Newton step. Let us also notice that the reduction to piecewise constant approximation at two adjacent cells provides a shock at the interface rather than at the interior point of the cell, so that the flux extremum can be detected and correctly approximated.

The convergence history on a sequence of uniform grids is plotted in fig.3.2b for polynomial degree $K \geq 0$. The $L_{1}$ - norm of the solution error,

$$
\|e r r\|_{L_{1}}=\int_{0}^{1}\left|U(x)-u_{h}(x)\right| d x,
$$


is computed in the regions where the solution is smooth. The error norm is shown in the logarithmic scale. An important feature of the suggested approach is that the polynomial degree of the approximate solution is only reduced, if necessary, for a transient solution at the current Newton step. Once the DG solution is correct (i.e. it is not "phantom" on a given cell), the original polynomial degree will be restored on the cell at next iteration. This approach allows one to obtain the optimal order of the convergence for high order DG discretizations.

Finally, let us notice that, while one should distinguish between two types of the solution overshoots arising in steady state problems, the solution oscillations due to inconsistent flux approximation at the flux extremum points are certainly more dangerous from a convergence point of view. It has been shown in the paper that the incorrect flux approximation impacts on the iterative method used in the problem (e.g. Newton's method) resulting in oscillations that cannot be eliminated by local limiters and a divergent solution. Thus, in our opinion, the methods of flux approximation originally developed for hyperbolic equations require further revision when steady state problems are considered.

Acknowledgments. This research was supported by The Boeing Company under contract 104AE. The author would like to thank F.T. Johnson and V. Venkatakrishnan for encouragement and fruitful discussions.

\section{REFERENCES}

[1] J. D. Anderson, Jr., Fundamentals of Aerodynamics, McGraw-Hill, New York, 1991.

[2] S. Balay, W. Gropp, L. C. McInnes and D. Smith, PETSc 2.0 Users Manual, Technical Report ANL 95/11, Argonne National Laboratory, 1997. http://www.mcs.anl.gov/petsc/petsc.html

[3] A. Burbeau, P. Sagaut and Ch. H. Bruneau, A problem - independent limiter for high - order Runge-Kutta discontinuous Galerkin methods, J. Comput. Phys., 169, 111-150, 2001.

[4] B. Cockburn, Discontinuous Galerkin methods for convection - dominated problems, in highorder discretization methods in computational physics, Lecture Notes in Comput. Sci. Engrg., Springer-Verlag, Heidelberg, 9, 69-224, 1999.

[5] B. Cockburn, G. E. Karniadakis and C. W. Shu, The development of discontinuous Galerkin methods, in Discontinuous Galerkin Methods. Theory, Computation and Applications, Lecture Notes in Comput. Sci. Engrg., Springer-Verlag, New York, 11, 3-50, 2000.

[6] B. Cockburn and C. Shu, The Runge - Kutta discontinuous Galerkin method for conservation laws V, J. Comput. Phys., 141, 199 - 224, 1998.

[7] V. Dolejší, M. Feistauer and C.Schwab, On some aspects of the discontinuous Galerkin finite element method for conservation laws, Mathematics and Computers in Simulation, 61, 333$346,2003$.

[8] B. Engquist and S. Osher, One-sided difference equations for nonlinear conservation laws, Math. Comp., 36, 321-352, 1981.

[9] C. Hirsh, Numerical computation of internal and external flows, John Wiley \& Sons, 2, 1990.

[10] H. Hoteit et al, New two-dimensional slope limiters for discontinuous Galerkin methods on arbitrary meshes, INRIA report, INRIA Rennes, France, 4491, 2002.

[11] D. S. Kershaw, M. K. Prasad, M. J. Shaw and J. L. Milovich, 3D unstructured mesh ALE hydrodynamics with the upwind discontinuous finite element method, Comput. Meth. Appl. Mech. Engrg., 158, 81 - 116, 1998.

[12] A. G. Kulikovskii, N. V. Pogorelov and A. Yu. Semenov, Mathematical Aspects of Numerical Solution of Hyperbolic Systems, Monographs and Surveys in Pure and Applied Mathematics, Chapman and Hall/CRC, Boca Raton, Florida, 188, 2001.

[13] R. J. LeVeque, Numerical Methods for Conservation Laws, Birkhäuser Verlag, Basel, Switzerland, 1992.

[14] R. B. Lowrier, Compact higher-order numerical methods for hyperbolic conservation laws, $\mathrm{PhD}$ thesis, The University of Michigan, 1996. 
[15] N. B. Petrovskaya, Newton's method for high-order schemes: as good as it gets?, preprint N11, Inst. Appl. Math., Rus. Ac. Sci., 2004.

[16] Y. Saad, Iterative Methods for Sparse Linear Systems, PWS Publishing Co., Kent, UK, 1995.

[17] A. M. Sorokin and N. A. Vladimirova, Anisotropic adaptation of unstructured volume grids to numerical solutions of three - dimensional gas dynamics problems, in Proceedings of the Workshop "Grid Generation: Theory and Applications", Moscow, 2002.

[18] Y. Sun and Z. J. Wang, Evaluation of discontinuous Galerkin and spectral volume methods for conservation laws on unstructured grids, AIAA, 2003-0253, 2003.

[19] J. J. W. van der Vegt and H. van der Ven, Space - time discontinuous Galerkin finite element method with dynamic grid motion for inviscid compressible flow, 33rd Computational Fluid Dynamics Course "Novel methods for solving convection dominated systems", the von Karman Institute, Rhode-St-Genese, Belgium, March 24-28, 2003.

[20] V. Venkatakrishnan, One-dimensional advection test problem, BCAG technical report, The Boeing Company, M/S 67-LF, Seattle, WA, 2002.

[21] V. Venkatakrishnan, S. Allmaras, D. Kamenetskii and F. Johnson, Higher order schemes for the compressible Navier-Stokes equations, AIAA, 2003-3987, 2003. 